\title{
Multipath Reliable Routing Using Cluster Based Communication
}

\author{
Dr. Shanti Rathore ${ }^{1}$, Nitesh Kumar Sharma ${ }^{2}$, Dr. M. R. Khan ${ }^{3}$ \\ ${ }^{1}$ Electronics and Communication Engineering Dr.C.V. Raman University Bilaspur, India \\ ${ }^{2}$ Electronics and Communication Engineering Dr.C.V. Raman University Bilaspur, India \\ ${ }^{3}$ Electronics and Telecommunication Engineering Government Engineering College Jagdalpur, India \\ ${ }^{1}$ rathoreshanti@gmail.com, ${ }^{2}$ Sharma786.nitesh@gmail.com, ${ }^{3}$ mrkhan@gecjdp.ac.in
}

Article History: Received: 11 January 2021; Accepted: 27 February 2021; Published online: 5 April 2021

\begin{abstract}
In this research we proposed the protection in ANT Optimized primarily based multipath congestion routing performance. Here the situation of DDoS is simulated and examines their impact in dynamic network. The multipath protocol like AOMDV is balance the all load by way of offering alternative path however not knowledgeable at each and every condition. The DDoS attacker is blocking off the whole viable direction in community by way of flooding large quantity of redundant packets in dynamic network. The attacker is that the intermediate node and this attacker contamination is continuously dispersing infection and the entire network overall performance is dumped. The proposed security scheme is identified attacker and their loss effect. Attacker is utterly disabled by way of proposed protection mechanism and their loss is also evaluated. The proposed approach is now not only detecting but also stop community from DDoS attack. The overall performance of protection scheme and assault is measured in three distinctive scenarios of a range of node densities. The proposed scheme is offers attacker free routing and get better network performance after making use of it. The performance of ANT OPTIMIZED is almost equal. The packets receiving, throughput, and PDR are bettering but the loss of packets and unnecessary flooding is decreased in dynamic network.
\end{abstract}

Keywords: MANET, AOMDV, ANT Optimized, DDoS, Security,Routing 2008

\section{Introduction}

Mobile ad hoc network is a sequence of all cellular nodes forming an advert-hoc community without the help of any centralized systems. These networks delivered a brand new architecture of networking fiction, it could be nicely outfitted to an surroundings the region each the infrastructure is misplaced or in which installation an all infrastructure isn't the excessive vary of the powerful value. The popular ieee 802. 11 "wireless" protocol is able to imparting advert-hoc network amenities at low degree, when no get entry to component is to be had. However, in this lookup case, the nodes are restrained to ship and accumulate records however do not route something during the community. Cell advert-hoc networks can feature in a standalone fashion or should perhaps be connected to a bigger network including the net [1]. Cellular ad hoc networks [2],[3] can turn the dream of having related "everywhere and at any time" into fact. Here common software program examples embody a catastrophe restoration or an operation. Now not observed in specific conditions, these networks may also additionally equally showcase better overall performance inside the exclusive places. For instance, we will reflect on consideration on a bunch of all peoples with laptops, in a commercial enterprise, meeting at a location the region no network offerings are gift. Each it is easy to results easily network their machines with extra forming an ad-hoc network. This is one of ten one among a range of examples the place these networks also can in keeping with chance be used. Ad hoc on-demand multipath distance vector (aomdv) shares several traits with aodv [3]. It's supported the residence vector routing and makes use of hop-through-hop routing method moreover of aomdv additionally unearths routes on call for the use of a method of direction discovery. The number one distinction is that is the quantity of routes discovered in each direction discovery. Here aomdv, path request (rreq) transmission from the supply closer to the destination establishes multiple reverse paths each at intermediate nodes in addition to, the vacation spot. More than one course respond as the (rreps) traverse those opposite paths lower back to shape more than one ahead paths to the vacation spot at the source stop to subsequent intermediate nodes. Right here cited the aomdv additionally affords intermediate nodes with alternate paths as they're determined to be useful in decreasing route discovery frequency [4]. Aomdv depended on the routing records already on hand in the underlying aodv set of rules, thereby restricting the overhead incurred in discovering a couple of paths. It doesn't hire any unique control packets. In fact of all greater rreps and rerrs for multipath discovery and protection are alongside with some extra fields in routing manipulate all packets (i.e., rreqs, rreps, and rerrs) constitute the sole more in routing control all packets (i. E., rreqs, rreps, and rerrs) represent the only more overhead in aomdv relative to healthy with the aodv.

\section{CONGESTION AVOIDANCE}

All through the preliminary statistics switch phase of a tcp connection [5] the gradual begin set of rules is used. However, there may want to even be a few quantity for the duration of gradual begin that the network is pressured to drop one or more packets thanks to overload or congestion. If this occurs, congestion avoidance [6] 
is used to slow the transmission fee. Even display, gradual start is utilized in routing conjunction with congestion avoidance due to the fact the functionality to induce the info switch going again started so it doesn't bog down and live slow. In the congestion could be avoidance set of rules a retransmission timer expiring or the reception of replica statistics acks can implicitly signal the sender that a network congestion the situation is taking location there after the sender nodes are right now devices its transmission home home windows to at least one half of the prevailing window measurement (the minimum of the congestion window and thus the receiver's advertised window length), however to no less than two segments. If there's a few congestion turned into as soon as indicated through a timeout, the congestion window is reset to at least one segment, which routinely puts the sender into gradual begin mode. If in congestion become as soon as indicated via way of duplicate acks, the quick retransmit and speedy healing algorithms are invoked (see below). Due to information is acquired within the course of congestion avoidance, the congestion window is expanded. However, gradual start is actually spent to the halfway point are occurring the area congestion at the beginning. Previously this midway point used to be recorded due to the fact the brand new transmission window. After this halfway factor, the congestion window is prolonged by way of one phase for all segments inside the all transmission home home windows which can be mentioned. This mechanism will pressure the sender to greater slowly develop its transmission price, because it's going to strategies the purpose the place congestion had in particular has been detected.

\section{ANTCOLONY OPTIMIZATION IN ROUTING}

ACO routing algorithms take notion from the behavior of ants in nature and from the associated area of aco to resolve the trouble of routing in conversation networks. Here the most sources of inspiration are discovered inside the potential of sure kinds of ants (e. G. Inside the family of argentine ants linepithemahumile) to discover the shortest route between their nest and a meals source the usage of a risky chemical substance known as pheromone. Ants travelling among the food supply and the nest leave lines of pheromone as they circulate. They also preferentially accompany inside the route of excessive pheromone intensities. In view that all of the shorter paths are regularly completed faster, they acquire higher levels of pheromone formerly because the viable attracting greater ants, which in flip cause greater pheromone. Here the all this amazing reinforcement technique permits the colony as a whole to converge at the shortest course course. This paperwork the idea of most of the upload the zone of aco [7].

\section{LITERATURE REVIEW}

A multipath hybrid routing set of rules for mobile ad-hoc networks. This algorithm is based on swarm genius algorithms and ant colony optimization (aco), mainly. By mapping arithmetic and all engineering problems on to organic societies, these strategies graph to solve the issues. Within the delivered aodv and aomdv set of rules, the amount of friends of a node has been looking to pick out subsequent hop [8]. A singular routing protocol primarily based on an improved ant colony optimization (aco) set of rules. The set of rules concentrates on the grant of qos and balanced strength electricity-intake over the all networks. With the introduction and basically of some metrics similar to the minimal path power and path hop matter quantity and with the aid of functionality of advancing a pheromone trail mannequin of the ant colony device, right here the set of rules innovatively gives heuristic ways respectively, primarily based on the duration or distance and the comfort of path to fulfill the excellent performance requirements of actual time and common traffics. Aco- aomdv is [10]. The authors existing here an ant colony optimization and advert-hoc on-call for multipath distance vector (aomdv) based totally routing protocol (acoaomdv) for ad hoc networks. In aco-aomdv, ant packets, savings simulated pheromone as a characteristic of multiple [9]. The author offers right here an ant colony optimization and ad-hoc on-call for multipath distance vector (aomdv) based completely routing protocol (acoaomdv) for unplanned networks. In aco-aomdv, ant packets, credit score simulated pheromone as a function of more than one parameters just like the understanding collected each and every direction visited, like average link depend of the direction, not unusual load of the direction, hop remember and therefore the present day pheromone the through nodes possess then on and furnish the know-the way to the traveling nodes to replace their pheromone tables with the aid of endowing the above, different types parameters like one among a type facts with brilliant load or weight values. [10]. A hybrid qos routing algorithm which will enhance the performance in manet is proposed with the useful resource of the hybrid fine of the algorithm makes it suitable for the environments in comparison with all reactive and proactive protocols. There ant's pheromone replace manner tactics has inherited advantages of robustness and fast convergence, which could makes it an suitable choice over present qos algorithms to give a boost to the performance for manet. Broaden an extended routing set of rules for manets supported ant colony optimization (aco) inspired with the necessary ants. There ant's pheromone update way methods have inherited advantages of robustness and short convergence, that could make it the appropriate preference over current qos algorithms to enhance the general performance for manet. Develop an advanced routing set of rules for manets supported ant colony optimization (aco) inspired with the crucial ants. The general performance of the routing set of rules is in evaluating via simulation and is as compared to an existing file manet routing protocol, unplanned 
on-demand distance vector (aodv). Even though average overall performance metrics are taken into consideration via excellent in situations with varying mobility levels and location traffic load [12].

\section{Methodology}

Mobile ad-hoc network are type of all through collection of nodes whose work, like an agent in between the sender to receiver node. Through the related research papers we determined it out the lookup hassle and optimized. During this paper, we diagram an more desirable most desirable protocol that more suitable the safety measures of MANET and provide congestion free verbal exchange below the various occasions or shape network. So here proposed security and congestion control mechanism used for inherited thru AOMDV and Ant colony optimization mechanism, because these strategies help to are looking for out fine nearer suitable multiple direction from supply to destination and provide reliable communication. Ad-hoc on demand multipath distance vector routing useful if quite one route is wished for the communication, its presents load balancing facility to the complete network, which will opt for three great shortest course out of all the handy paths, but it's no longer suitable for all pairs of communication so in our proposed method we tune and strategies or optimized multipath decision via Ant colony optimization (ACO) methodology.

\section{DDOS Prevention}

Distributed denial of service attack or trade the quite a number network behaviors thanks to network node atypical performance so DDOS attack is generate a couple of deceptive behavior of community i.e. routing, format, spoofing, etc. our proposed Ant base safety mechanism will supply lightweight safety method and extra appropriate for dynamic network, due to the fact we use tune the network through its pheromone behavior, the nodes pheromone is really helpful to detection of DDOS nodes so it's in the future can't enter the junk message generated by using a DDOS attacker inside the network and minimized the congested environment. DDOS attacker node spread the junk message to the susceptible node the usage of the handshake technique and captures it, that message ahead to subsequent hop and after a short duration of someday complete community crashed suddenly.

Algorithm: DDOS Detection and Prevention

Input:

M: mobile nodes

S: sender nodes

$\mathrm{R}$ : receiver nodes

I: Intermediate nodes

$\Psi=$ radio range AOMDV: routing

Si: Suspicious nodes higher reachable

Endif

SendacknowledgetoSusingreversepath

$\operatorname{Data}(\mathrm{S}, \mathrm{R}, \mathrm{I})$

Else

DDOSdetection/prevention

PwatchInodes

If(Isendjunkmessage \&\&m 1 receives)

Then

m1 $\square$ congested

Else

Networkforwarddatethroughnormalpaths

UseACObyallMnodes

Calculatenew_phofInodes $\square$

(Forward/receives) \pm old_ph

ClassifyInodebyfzvalue

Updateroutethroughnew_phvalue

Endif

Pexecutedetectionmodule

thenSetIasSi

If(Isendjunkmessage \&\&pkt!=normal)

$\mathrm{Si} \square$ receives\&\&notforward

$\mathrm{A} \square \mathrm{Si}$

DetectbyPpackettypeofA,

Timeofattack

Nodenumber

Numberofjunkmessage 


\section{Endif}

Pexecutepreventionmodule

Ifnodedetectjunkspreader\&\&A $\square$ DDOSthen

Aph $\square 0$

$\mathrm{P} \square$ broadcastnodeAasattacker

$\mathrm{P} \square$ blockAnode

\section{Endif}

\section{SIMULATION PARAMETER}

The simulation of the previous and proposed protocol is simulated on this work foundation of these regarded parameters. These parameters are frequent right here in DDoS attacker presence and security scenario.

A:Attackernodes

P:Preventernode

O:Optimizationtechnique(ACO)

$\mathrm{f}_{\mathrm{Z}}=\{0.0,0.1,0.2, \ldots \ldots \ldots .1 .0\}$

$\mathrm{PH}=\sum_{p=0}^{1} \mathrm{hp} 0 \mathrm{hpi}$

Output:TCP,UDP, attacker Percentage,Attacker node identification

Procedure:

$\operatorname{AOMDV}(\mathrm{S}, \mathrm{R}$, route_pkt, $\Psi)$

If(Iin $\Psi$ andI!=R)then

$\mathrm{I} \square \operatorname{setph}(0$ to 1$)$ forwardingcriteria

$\mathrm{I} \square$ forwardroute_pkt

Elseif(I==R\&\&path>1)then

Select best three paths whose ph value

Node out of range or not receiver

\section{5 results and discussion}

The effects analysis via assessment and dialogue is noted at some stage in this section that measures the performance in the presence of DDoS assault and safety $\mathrm{m} 1 \square$ not forward genuine pkt $\mathrm{m} 1 \square$ broadcast junk message to $\mathrm{M}$ capture all $\mathrm{M}$ nodes Network congested

\section{Table1.5SimulationParameters}

\begin{tabular}{|c|c|}
\hline Parameters & Type \\
\hline Network Type & M50ANET \\
\hline Mobile Nodes & $10,30,50$ \\
\hline Physical Medium & Wireless Physical \\
\hline Propagation Modes & Two ray Ground \\
\hline Antenna Type I & Omni Directional Antenna \\
\hline Simulation Area & $800 * 800 \mathrm{~m} 2$ \\
\hline Simulation Time & 100 Seconds \\
\hline Frequency & $914 \mathrm{e}+6 \mathrm{Mhz}$ \\
\hline MAC Layer & 802.11 \\
\hline Routing Protocol & AOMDV ANT \\
\hline Attack Type & DDOS \\
\hline Prevention & Message Identification \\
\hline Queue Type & Drop Tail / Priority Queue \\
\hline
\end{tabular}

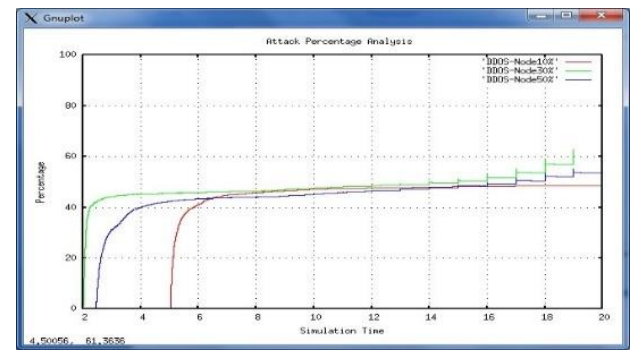

Fig.1AttackerLossAnalysis 


\section{A. Attacker Percentage Analysis}

The DDoS attacker for the duration of this community flooding undesirable packets and these packets aredegrading all coming community overall performance by means of blocking off hyperlink capacity. The attacker nodes are additionally infected other all nodes that function the identical things to do in the community as the attacker. During this research graph the drop thanks to attacker presence community is measured altogether three situations of varied node density. The facts losing in quite a few node densities are getting into being a special, but the proposed security scheme is prevent community from malicious effect of attacker and presents impervious and secure routing. The attacker infection is accelerated with reference to time and most drop share reaches 60 to $62 \%$. The attacker drop percentage is definitely removed from community after making use of security scheme in the nonlinear dynamic network.

\section{B. Attack Detection in 10 nodes Scenario}

Here the DDoS attacker is flooded all unwanted packets in community and these packets don't include any useful records due to that these packets are only flooded in network for ingesting confined vary of bandwidth capacity. Within the DDoS attacker is spread their malicious behavior, there used to be some particular and due to attacker contamination other nodes are additionally behaving like an attacker. There In some characterized desk 1, table two and table 3 the distinct situation displaying of the node density is noted and recognized that the amount of attacker's extent is one of a kind in the one of a kind node density scenario. In 10 node density only node 4 is no longer flooded first-rate deal and their contamination is additionally very minimum. In situation of 30 nodes and 50 nodes huge quantities of packets are flooded with many nodes.In 10 nodes situation quantity of attackers are 6 nodes, in 30 node situation quantity of attackers are 8 nodes and in it 50 cellular node scenario wide variety of attackers are 7 nodes in dynamic networks.

Table1AttackerAnalysisin10nodedensity

\begin{tabular}{|c|c|c|}
\hline $\begin{array}{c}\text { DDOS } \\
\text { Attacker }\end{array}$ & $\begin{array}{c}\text { Packet } \\
\text { Spread }\end{array}$ & $\begin{array}{c}\text { Percentage of } \\
\text { Infection }\end{array}$ \\
\hline 2 & 59828 & 11.26 \\
\hline 3 & 50410 & 9.49 \\
\hline 4 & 589 & 0.11 \\
\hline 5 & 47945 & 9.02 \\
\hline 6 & 55481 & 10.44 \\
\hline 7 & 43843 & 8.25 \\
\hline
\end{tabular}

Table2AttackerAnalysisin30nodedensity

\begin{tabular}{|c|c|c|}
\hline $\begin{array}{c}\text { DDOS } \\
\text { Attacker }\end{array}$ & $\begin{array}{c}\text { Packet } \\
\text { Spread }\end{array}$ & $\begin{array}{c}\text { Percentage of } \\
\text { Infection }\end{array}$ \\
\hline 8 & 67850 & 6.21 \\
\hline 9 & 43567 & 3.99 \\
\hline 12 & 185710 & 17.01 \\
\hline 15 & 43904 & 4.02 \\
\hline 17 & 55517 & 5.08 \\
\hline 20 & 41416 & 3.79 \\
\hline 21 & 67614 & 6.19 \\
\hline 29 & 178515 & 16.35 \\
\hline
\end{tabular}

Table3AttackerLossin50nodedensity

\begin{tabular}{|c|c|c|}
\hline $\begin{array}{c}\text { DDOS } \\
\text { Attacker }\end{array}$ & $\begin{array}{c}\text { Packet } \\
\text { Spread }\end{array}$ & $\begin{array}{c}\text { Percentage of } \\
\text { Infection }\end{array}$ \\
\hline 2 & 45218 & 6.06 \\
\hline 7 & 66841 & 8.95 \\
\hline 24 & 39303 & 5.26 \\
\hline 27 & 128547 & 17.21 \\
\hline 32 & 42770 & 5.73 \\
\hline 34 & 6404 & 0.86 \\
\hline 36 & 70138 & 9.39 \\
\hline
\end{tabular}




\section{Conclusion}

This should clearly explain the main conclusions of the work highlighting its importance and relevance. In this research the likelihood of attacker existence is extra if the flooding by means of packets is always uncontrolled and continuously increases with reference to time. The proposed protection scheme is utilized on ACO (Ant Colony Optimization) pheromone based totally multipath to secure network via the use of the DDoS attack. The attacker is detected with the aid of the heavy flooding of all routing packets and these packets flooded via the node are addressed via a security scheme to dam it permanently exact in the course of this network. Here the effect of the proposed scheme is that the processing functionality of nodes is utilized for max facts forwarding to next neighbor route to the appropriate and receiving response from a neighbor or sender node.The proposed scheme is far higher to impervious all cellular networks from hazardous DDoS attacks. The performance of the community is a measure of the three distinct node density situation and outcomes are of course existing in result the robust impact of the proposed scheme through bettering packets receiving and removes the flooding impact of the attacker. The presence of proposed security mechanism is lowering packet loss and flooding and improves throughput and PDR performance in dynamic network with sending and receiving data output.

\section{Future scope}

In future we also measure the some greater performance analysis of the proposed protection scheme with electromagnetic in transport nano network layer protocol. The TCP and UDP are the transport layer protocol and their performance in term of packet loss measures and frequency bandwidth.In future we also measure the some extra performance analysis of the proposed security scheme with electromagnetic in transport nano network layer protocol. The TCP and UDP are the transport layer protocol and their performance in term of packet loss measures and frequency bandwidth.

\section{References}

1. Tasman Networks Inc. Routing basics :(2004) Protocol evolution in enterprise and service provider networks. Technical report.

2. Yang H., Luo H., Ye F., Lu S. and Zhang L 2004). Security in Mobile Ad Hoc Networks: Challenges and Solutions,IEEE Wireless Communications, 11(1), pp.38-47.

3. Mahesh K.Marina, Samir R. Das (2006),Ad hoc On-Demand Multipath Distance Vector Routing ,Wireless Communications And Mobile Computing,Publishedonlinein Wiley Inter Scienc(www.interscience.wiley.com) 6,969-988.

4. JingyuanWang,Jiangtao Wen et. al.(2011)An Improved TCP Congestion Control Algorithm and its Performance IEEE.

5. KMakoto Ikeda, Elis Kulla, Masahiro Hiyama, Leonard Barolli, Rozeta Miho and Makoto Takizawa(2012,Congestion Control for Muli-flow Traffic in Wireless Mobile Ad-hoc Networks, IEEE Sixth International Conference on Complex, Intelligent, and Software Intensive Systems.

6. M. Subha and Dr.R.Anitha(2009) An Emerging Ant Colony Optimization Routing Algorithm (Acora)For MANETs,Journal of Computer Applications, Vol.2 No.3

7. Mohammad Golshahi,MohammadMosleh and Mohammad Kheyrandish(2008)Implementing an ACO Routing Algorithm for ADHOC Networks ,Proceeding of the IEEE International Conference on Advanced Computer Theory and Engineering.

8. S.Kannan,T.Kalaikumaran,S.Karthik and V.P. Arunachalam(2010) Ant Colony Optimization for Routing in Mobile Ad-Hoc Networks,International Journal of Soft Computing,5(6), pp.23-228.

9. Xun-bing Wang, Yong-zhao Zhan, Liang-min Wang and Li-ping Jiang(2008) Ant Colony Optimization and Ad-hoc On-demand Multipath Distance Vector(AOMDV)Based Routing Protocol, Proceedings of the Fourth International Conference on Natural Computation.

10. Singh Rajeshwar,Singh D K andKumarLalan Kumar(2010) Ants Pheromone for Quality of Service Provisioning In Mobile Ad hoc Networks, International Journal of Electronic Engineering Research, 2(1), pp.101-109.

11. EseosaOsagie,ParimalaThulasiraman and RuppaK.Thulasiram(2011) PACONET improved Ant Colony Optimization routing algorithm for mobile ad hoc NETworks,IEEE Computer Society 22nd International Conference on Advanced Information etworking and Applications,pp.204-211 